\title{
A Computational Technique for Iron Losses in Electrical Machines
}

\section{INTRODUCTION}

Designing and analyzing an electrical machine can be complicate due to the complex geometry and the nonlinear materials. In particular two important aspects have to be taken into consideration during the design and analysis process: accuracy and computational time [1]. Many analytical models [2]-[4] have been developed. Such models are very useful for an initial design, but they cannot be adopted for accurate representation of the machine due to complex geometric shapes and nonlinear materials. Therefore, Finite Element (FE) models have to be considered at the expense of computational time, mostly if the model is considered 3-Dimensional. The goal is thus to improve the accuracy of the model reducing the computational time.

An example can be found in the computation of the iron losses of an electrical machine. Often, the iron losses are computed in post-processing and they are estimated by means of empirical formulas which are not precise. Calculating them explicitly requires long execution time because a 3D model is required. In order to avoid this inconvenient, the iron losses have to be modeled considering particular approaches such as homogenization techniques and using vectorized hysteresis models, both applied to 2D FE models.

In this paper, a new computation method for the iron losses in electrical machines is adopted taking into account the minimization of the computational time through the implementation of a homogenization technique. In particular, the iron losses due to the eddy currents are modeled either explicitly or adopting a homogenization technique, while the iron losses due to the hysteresis phenomenon are modeled incorporating a Jiles-Atherton vector hysteresis model. In order to validate the model, an iron ring available in our laboratory has been considered in order to perform some experimental tests. Several FE simulations have been carried out highlighting the accuracy of the homogenization technique and of the hysteresis model. The experimental and numerical results will be compared in the full paper. The considered model will be applied to an inset Permanent Magnet (PM) synchronous machine.

\section{IRON LOSSES MODELS}

The losses in iron laminations can be split into three parts: eddy current losses, hysteresis losses and excess losses as reported in (1) [5].

$$
P_{f e}=P_{e d d y}+P_{h y s}+P_{e x c}=k_{e d d y} f^{2} B_{m}^{2}+k_{h y s} f B_{m}^{\alpha}+P_{e x c}
$$

where $f$ is the frequency, $B_{m}$ the peak value of the magnetic flux density, $k_{e d d y}, k_{h y s}$ and $\alpha$ are constants provided by the manufactures. This standard method is typically implemented in the most part of the available FE software, nevertheless it is not precise because it assumes sinusoidal variation of the magnetic flux density and it depends from empirical parameters provided by the manufacture of the iron lamination. Other more accurate approaches have to be adopted in order to overcome the limit of the standard method [1]. In next sections, new method for the computation of the iron losses will be explained.

\section{A. Iron losses due to eddy currents}

An iron lamination of width $w$, length $l$ and thickness $d$ is considered. The lamination is parallel to the $x z$-plane and the middle of the thickness $d$ coincides with $y=0$. The iron is assumed magnetically isotropic and its electrical conductivity $\sigma$ is constant. The lamination carries a magnetic flux density $\mathbf{B}$ that can be induced by permanent magnets as well as windings.

In order to compute the eddy currents induced in the lamination, the following differential equations and constitutive laws can be considered:

$$
\begin{gathered}
\nabla \times \mathbf{E}=-\frac{\partial \mathbf{B}}{\partial t}, \quad \nabla \times \mathbf{H}=\mathbf{J}, \quad \nabla \cdot \mathbf{B}=\mathbf{0}, \\
\mathbf{J}_{e d d y}=\sigma \mathbf{E}, \quad \mathbf{H}=\nu\left(\mathbf{B}^{2}\right) \mathbf{B},
\end{gathered}
$$

where $\mathbf{E}$ is the electrical field, $\mathbf{B}$ the magnetic flux density, $\mathbf{H}$ the magnetic field and $\mathbf{J}_{\text {eddy }}$ the eddy current density. Moreover, the reluctivity $\nu=\mu^{-1}$ is a function of $\mathbf{B}^{2}$.

In order to compute the eddy current distribution, a 3D FEA has to be considered, but it requires a high computational time. Thereby, 2D FEA are considered neglecting the 3D effects in the lamination. There are different ways for 


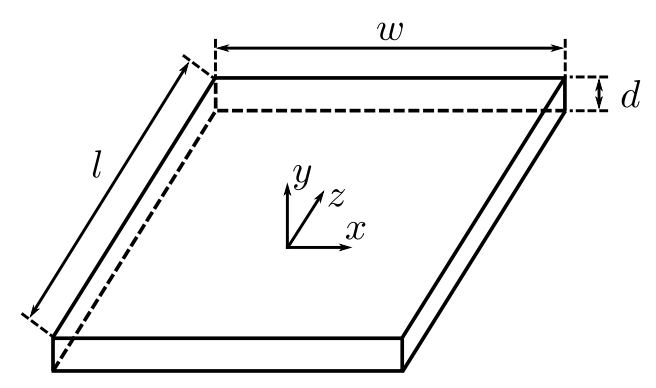

Fig. 1. Iron lamination of width $w$, length $l$ and thickness $d$.

computing the iron losses due to eddy currents: either explicitly implementing the (2) or considering a nonlinear homogenization technique. However, particular attention has to be paid when (2) are explicitly implemented. In particular, the homogenization technique allows to implement 1D problems coupled with the FE equations approximating, in this case, the behaviour of the fields in the lamination and reducing the computational time. To apply that homogenization technique, the equations described in [6] have to be coupled in the formulation of the field. From the values obtained by the formulation resolution, it is possible to compute the iron losses applying the homogenization technique along $y$-axis in the post-processing. In our case a II-order homogenization has been considered and it means the magnetic flux density is approximated with a II-order polynomial as function of $y$.

\section{B. hysteresis losses}

The Jiles-Atherton model is used to determine the iron losses due to the hysteresis phenomenon [7]. The full description of the hysteresis loss inclusion will be presented in the full paper.

\section{VALidATION OF THE MODEL}

The method described above has been implemented end validated considering two machines: an iron ring and an inset PM synchronous motor. In the first case, the iron ring has been simulated considering both 2D and 3D models which have been validated by experimental results. A comparison between the standard method and the new approach will be presented in the full paper. In the second case, the method is applied either to a $2 \mathrm{D}$ geometry or 3D geometry.

\section{A. Description of the iron ring machine}

As said above, an iron ring available in our laboratory is considered and it is shown in Fig. 2. It is characterized

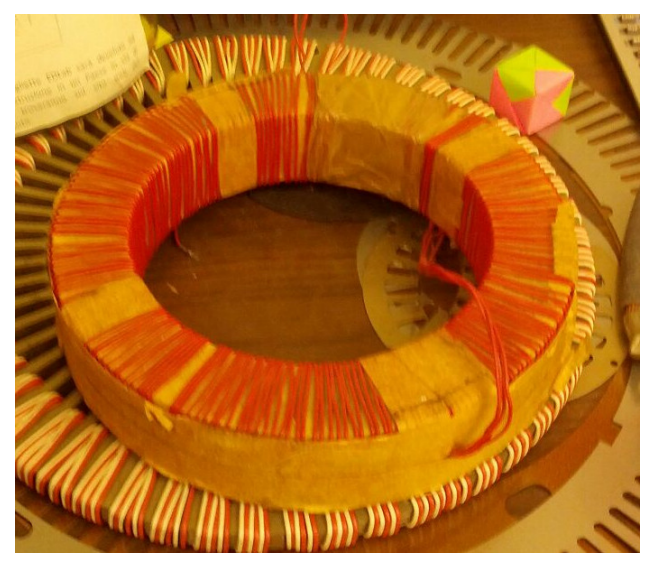

Fig. 2. The iron ring simulated by way of $2 \mathrm{D}$ and $3 \mathrm{D}$ FEA and tested by experimental tests.

by a laminated iron core composed by 80 laminations with an external diameter $D_{e}=215 \mathrm{~mm}$, an internal diameter $D_{i}=145 \mathrm{~mm}$ and a height $h=40 \mathrm{~mm}$. The two windings are obtained by 100 turns.

Such a machine is particular suitable for determining the iron losses because the magnetic flux density inside the iron is quite constant in a cross-section of the machine and the joule losses in the windings can be well determined. 


\section{B. Finite Element simulations and experimental results}

Several transient 2D and 3D FE simulations of the iron ring have been carried out considering different frequencies and loads. A sketch of the geometry of the iron ring is shown in Fig. 3. The simulated geometry is half of the whole cross section of the real machine. Basically, it is composed by the iron stack surrounded by the coil. Between the coil and the iron stack, a thin film of air is present. Another region of air surrounds the geometry until the external boundary where the constraints are applied. Between each lamination, a thin film of isolation is considered. The supply sources, both current and voltage, can be applied by means of external circuit coupling.

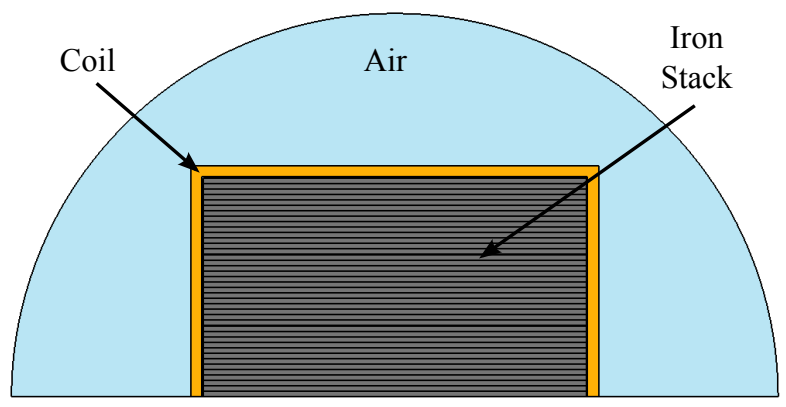

Fig. 3. A sketch of the geometry of the iron ring.

In the FE model, both eddy current and hysteresis phenomena are considered.

As said before, the distribution of the eddy currents in the laminations can be computed either explicitly or by way of homogenization technique. In the first case, the mesh in Fig. 4(b) is considered. The number of elements in the thickness $d$ is 10 and they are necessary for highlighting the skin effects. In the second case, Fig. 4(a) shows the mesh used when the homogenization technique is applied. The elements number in the thickness $d$ can be limited to two elements because the distribution of the eddy currents can be straightforwardly reconstructed in the post-processing.

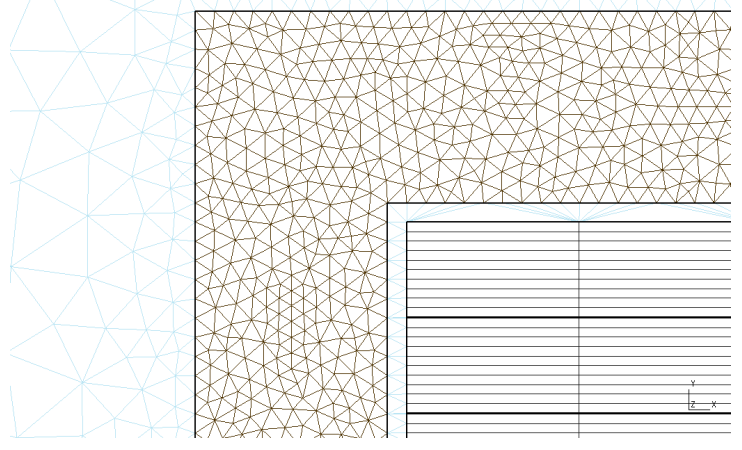

(a) mesh used for the explicity computation of the eddy current.

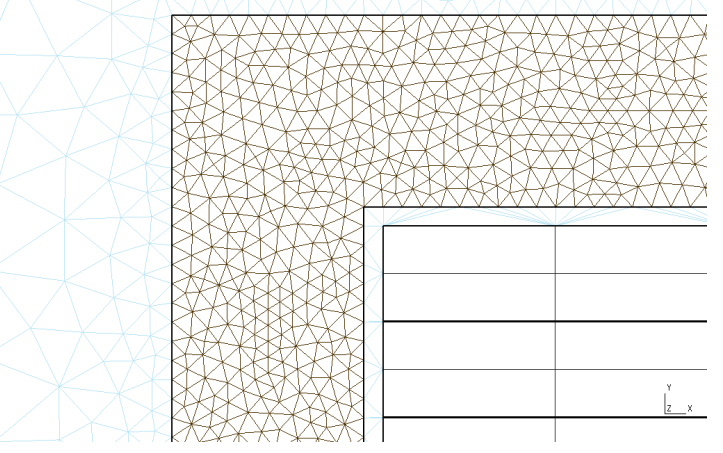

(b) mesh used with the homogenization technique.

Fig. 4. Details of the mesh used in the simulations.

In Fig. 5 the distribution of the eddy currents in the laminations is shown imposing a current density $J=10^{6} \mathrm{~A} / \mathrm{m}^{2}$ at frequency $f=5 \mathrm{kHz}$. A high value of the frequency has been chosen in order to highlight the skin effects in the laminations. Fig.5(a) shows the distribution when the eddy currents are explicitly computed, while Fig.5(b) shows the distribution when the eddy current are computed with the homogenization technique. It is worth noticing that no border effects are present using the homogenization technique due to the approximated model. For this comparison the mesh has been considered the same.

In Fig. 6 the distribution of the magnetic flux density $|\mathbf{B}|$ along a lamination thickness $d$ is reported either for the explicit computation or for the computation through homogenization technique. The label nohomo indicates the explicit computation, while the label homo the computation through homogenization technique. As can be noticed, the curve obtained with homogenization technique overlaps the curve obtained with the explicit computation. The curve of explicit computation is segmented due to the discretization of the laminations.

Fig. 7 shows the iron losses due to the eddy currents in a lamination of thickness $d$ versus the frequency. 2D model with homogenization technique and 3D model with explicit computation are considered and it is worth noticing a good agreement between the results. 


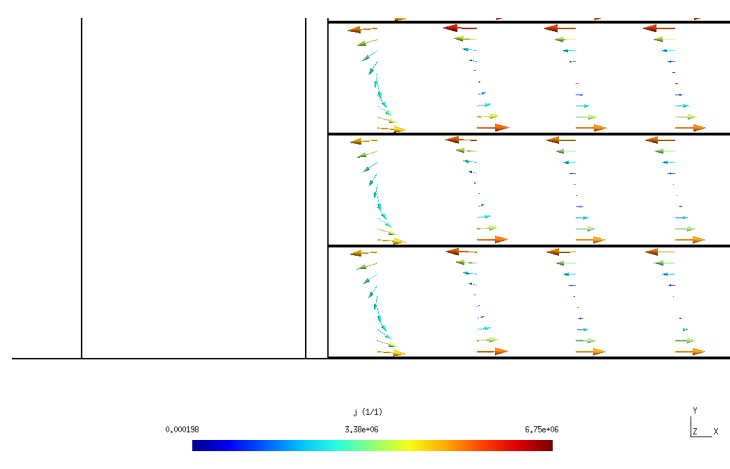

(a) explicit computation.

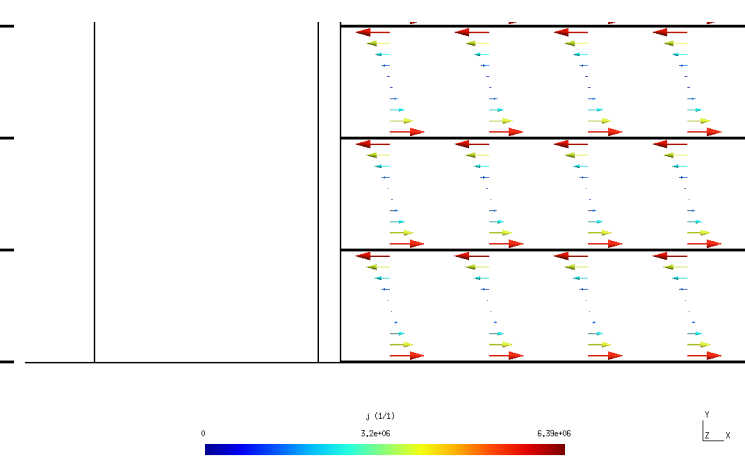

(b) homogenization technique.

Fig. 5. Details of the eddy current distribution.

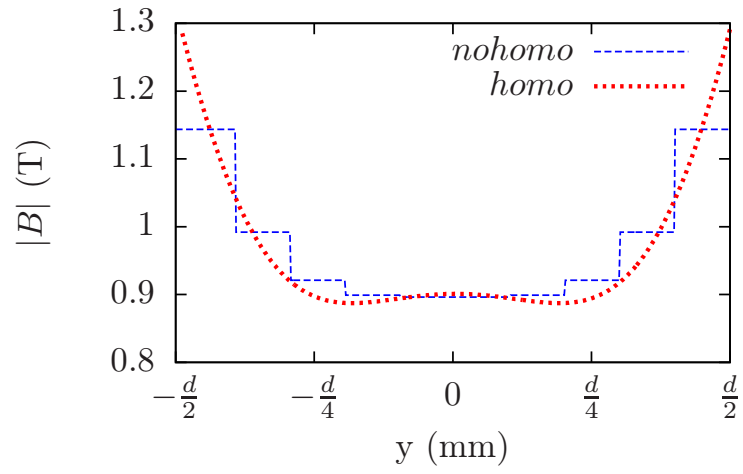

Fig. 6. Distribution of the magnetic flux density $|\mathbf{B}|$ in a lamination thickness $d$.

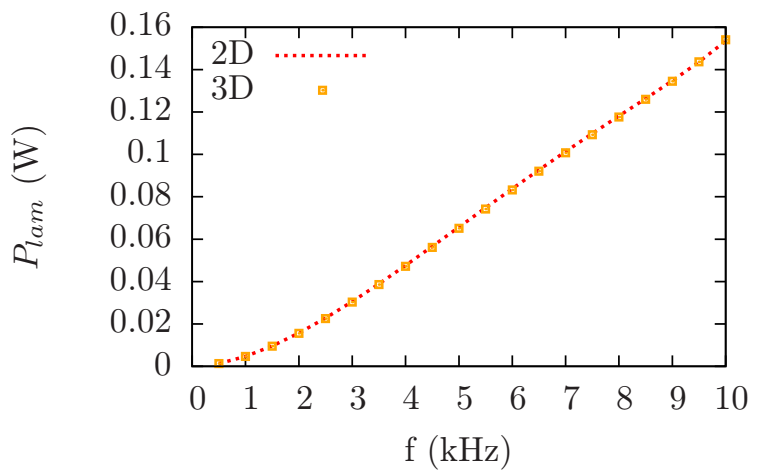

Fig. 7. Iron losses due to the eddy currents in a lamination vs the frequency. $2 \mathrm{D}$ model with homogenization technique and $3 \mathrm{D}$ model with explicit computation of the losses are considered.

More details on the hysteresis phenomenon, the comparison between standard and new method and the experimental results will be shown in the full paper.

\section{Application EXAMPle on A InSET PM SYNCHRONOUS MACHINE}

After the validation of the model through experimental tests, a particular inset PM machine is considered. This kind of machine is obtained starting from a Surface Permanent Magnet (SPM) machine and by adding a sort of iron teeth between each couple of magnets. In Fig. 8(a) a sketch of the geometry of the machine is shown, while in Tab. I the main data are reported. Fig. 8(b) and 8(c) represents the magnetic flux lines in the machines at no load and at full load conditions. The motor is simulated in 2D and 3D models considering eddy current losses and hysteresis phenomenon. In the 2D model, the homogenization technique for the computation of the eddy current losses is adopted [9].

More details will be reported in the full paper

\section{Conclusion}

In this paper, a new computation method for the iron losses in electrical machines is adopted taking into account the minimization of the computational time through the implementation of a homogenization technique. In particular, the iron losses due to the eddy currents are modeled either explicitly or adopting the homogenization technique, while the iron losses due to the hysteresis phenomenon are modeled incorporating a Jiles-Atherton vector hysteresis model. Several 2D and 3D FE simulations have been carried out in order to validate the computation method and a good agreement has been found in the results. The experimental and numerical results will be compared in the full paper. The considered model will be applied to an inset Permanent Magnet (PM) synchronous machine.

\section{REFERENCES}

[1] Sizov, G.; Ionel, D. \& Demerdash, N., "A review of efficient FE modeling techniques with applications to PM AC machines", 2011 IEEE Power and Energy Society General Meeting, 2011, pp. 1-6 


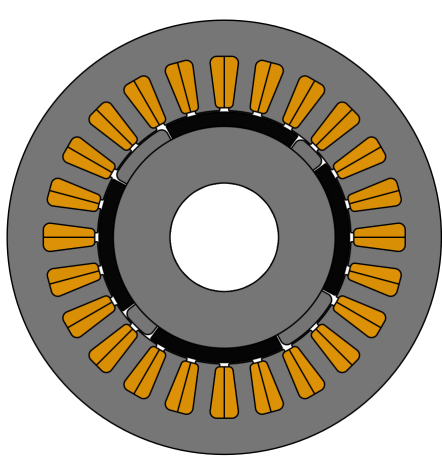

(a) sketck of the motor geometry

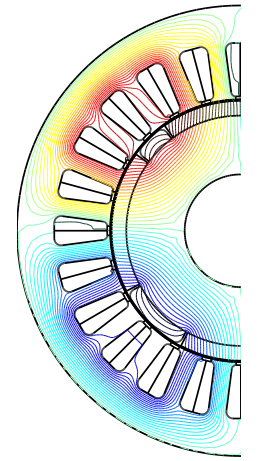

(b) flux lines at no load

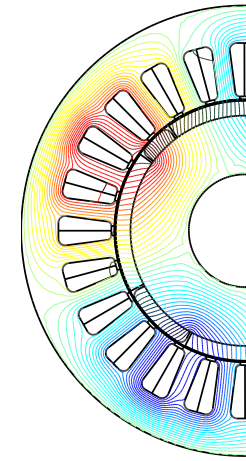

(c) flux lines at full load

Fig. 8. Cross-sections of the inset machine.

TABLE I

MAIN GEOMETRY AND MATERIAL DATA OF THE INSET MOTOR

\begin{tabular}{lll}
\hline Pole number & $2 p$ & 4 \\
Phase number & $m$ & 3 \\
Nominal Current & $I_{n}$ & $3 \mathrm{~A}$ \\
Phase resistance & $R_{p h}$ & $6.75 \Omega$ \\
Slot number & $Q_{s}$ & 24 \\
Fill factor & $k_{f i l l}$ & 0.5 \\
Air gap & $g$ & $0.3 \mathrm{~mm}$ \\
External diameter & $D_{e}$ & $120 \mathrm{~mm}$ \\
Inner diameter & $D_{i}$ & $70 \mathrm{~mm}$ \\
Slot height & $h_{s}$ & $15 \mathrm{~mm}$ \\
Back iron height & $h_{b i}$ & $10 \mathrm{~mm}$ \\
Stack length & $L_{s t k}$ & $40 \mathrm{~mm}$ \\
lamination thickness & $d$ & $0.65 \mathrm{~mm}$ \\
PM coercitive field & $H_{c}$ & $-629 \mathrm{kA} / \mathrm{m}$ \\
\hline & &
\end{tabular}

[2] Z. J. Liu, and J. T. Li, "Accurate Prediction of Magnetic Field and Magnetic Forces in Permanent Magnet Motors Using an Analytical Solution", IEEE Trans. on En. Conv., Vol. 23, No. 3, pp. 717-726, September 2008.

[3] D. Zarko, D. Ban, and T.A. Lipo, "Analytical Calculation of Magnetic Field Distribution in the Slotted Air Gap of a Surface PermanentMagnet Motor Using Complex Relative Air-Gap Permeance", IEEE Transactions on Magnetic, vol. 42, pp. 1828-1837, 2006.

[4] C. B. Rasmussen, and E. Ritchie, "A Magnetic Equivalent Circuit Approach for Predicting PM Motor Performance", IEEE Industry Applications Society Annual Meeting, New Orleans, Louisiana, October 5-8, pp. 10-17, 1997.

[5] Bertotti, G.; Boglietti, A.; Chiampi, M.; Chiarabaglio, D.; Fiorillo, F. \& Lazzari, M., "An improved estimation of iron losses in rotating electrical machines", IEEE Transactions on Magnetics, 1991, vol. 27, pp. 5007-5009

[6] Gyselinck, J.; Sabariego, R. \& Dular, P., "A nonlinear time-domain homogenization technique for laminated iron cores in threedimensional finite-element models", IEEE Transactions on Magnetics, 2006, vol. 42, pp. 763-766

[7] Gyselinck, J.; Dular, P.; Sadowski, N.; Leite, J. \& Bastos, J., "Incorporation of a Jiles-Atherton vector hysteresis model in 2D FE magnetic field computations: Application of the Newton-Raphson method", COMPEL - The international journal for computation and mathematics in electrical and electronic engineering, 2004, vol. 23, pp. 685-693

[8] Jiles, D.; Thoelke, J. \& Devine, M., "Numerical determination of hysteresis parameters for the modeling of magnetic properties using the theory of ferromagnetic hysteresis", IEEE Transactions on Magnetics, 1992, vol. 28, pp. 27-35

[9] Gyselinck Johan. Geuzaine, C. V. S. R., "Considering Laminated Cores and Eddy Currents in 2D and 3D Finite Element Simulation of Electrical Machines", 18th Conference on the Computation of Electromagnetic Fields (COMPUMAG2011), 2011, pp. 2

[10] Benabou A., C. S. \& Piriou, F., "Comperison of Preisach and Jiles-Atherton models to take into account hysteresis phenomenon for finite element analysis", Journal of Magnetism and Magnetic Materials, 2003, vol. 261, pp. 305-10

[11] Narita, K.; Asanuma, T.; Semba, K.; Sano, H.; Yamada, T.; Aiso, K. \& Akatsu, K., "An accurate iron loss evaluation method based on finite element analysis for switched reluctance motors", 2015 IEEE Energy Conversion Congress and Exposition (ECCE), 2015, pp. 4413-4417

[12] Bertotti, G., "General properties of power losses in soft ferromagnetic", IEEE Transactions on materials Magnetics, 1988, vol. 24, pp. 621-630

[13] Boglietti, A.; Cavagnino, A.; Lazzari, M. \& Pastorelli, M., "Predicting iron losses in soft magnetic materials with arbitrary voltage supply: an engineering approach", IEEE Transactions on Magnetics, 2003, vol. 39, pp. 981-989

[14] Kitao, J.; Hashimoto, K.; Takahashi, Y.; Fujiwara, K.; Ishihara, Y.; Ahagon, A. \& Matsuo, T. "Magnetic Field Analysis of Ring Core Taking Account of Hysteretic Property Using Play Model", IEEE Transactions on Magnetics, 2012, vol. 48, pp. 3375-3378

[15] Bottauscio, O.; Chiampi, M. \& Chiarabaglio, D., "Advanced model of laminated magnetic cores for two-dimensional field analysis", IEEE Transactions on Magnetics, 2000, vol. 36, pp. 561-573 